\title{
Biomimetic Design of Low Stiffness Actuator Systems for Prosthetic Limbs
}

\author{
D. L. Russell and M. McTavish \\ Department of Mechanical and Aerospace Engineering \\ Carleton University, Ottawa, Canada, \\ drussell@mae.carleton.ca
}

\begin{abstract}
The various relationships that are possible between the mechanical properties of single actuators and the overall mechanism (in this case a human arm with or without a prosthetic elbow) are discussed. Graphical and analytical techniques for describing the range of overall limb stiffnesses that are achievable and for characterizing the overall limb stiffness have been developed. Using a biomimetic approach and, considering energetic costs, stability and complexity, the implications of choosing passive or active implementations of stiffness are discussed. These techniques and approaches are particularly applicable with redundant (agonist - antagonist) actuators and multiple degrees of freedom. Finally, a novel biomimetic approach for control is proposed.
\end{abstract}

\section{Introduction}

From an engineering point of view, many would define an ideal actuator as one that generates the desired response (motion or force) accurately and immediately. Most would also insist that the response of the actuator be linear. In practice, real actuators do not meet this ideal generally due to a mismatch in dynamic characteristics or due to power limitations. Typical applications use feedback to attempt to modify the performance to approach the "ideal". Another important characteristic assumed of "ideal" actuators is that they reject disturbances. For example, a velocity actuator will achieve the desired velocity no matter what force is opposing it or a force actuator will generate the required force no matter how fast it is required to move. That this can depend significantly on the task is demonstrated by considering that It is very difficult to push on a unrestrained ping-pong ball with a large force. The relationship between the force and motion is summarized as the mechanical impedance of the device. In a simple case where the relationship is between force and position the impedance becomes a stiffness. The typical engineering approach is also to use one actuator for each degree of freedom and to arrange the design so that the degree of cross-coupling between different degrees of freedom is minimized.

In the biological world actuators are generally muscles and muscles are not in any of the above ways ideal. They are highly nonlinear and the relationship between the input "command" neural signals and the resulting changes in the muscle are highly complex. To further complicate the situation, muscles most often work in situations where they are redundant, that is where more than one muscle may accomplish a given objective. Muscles are unidirectional actuators and therefor generally work in opposition about a joint in agonist - antagonist pairs. Despite these numerous complexities, the human arm has capabilities and a general level of mechanical performance far in excess of any practical robotic replacement (a prosthetic limb). Prosthetic limbs can lift loads up to approximately 10 lbs and can move from one extreme to the other, approximately 135 degrees in about 1 second. The human arm can lift more, lift it faster and interact with complicated dynamic situations.

Our biomimetic approach to prosthetic limb (or robotic arm) design involves resolving design issues around the use of multiple, low impedance, nonlinear actuators acting around a joint or joints in a limb (or robotic arm). It is important to have a clear understanding of the complex relationship between the configuration of these actuators and the mechanical performance of the limb (or robotic arm). The results help to clarify our understanding of the mechanical issues in limb or robotic arm design and in understanding the biological solution to this design challenge - the human arm (see for example, Beer et al [1]). 


\subsection{Compliant Actuators}

Compliance (the inverse of stiffness) is present in all physical actuator systems as the mechanical components in the drive train deflect under the applied loads. The deflection may be entirely described as a relationship between load and displacement or the situation may be more complex including, for example, loads that depend on rates of displacement. An actuator may be considered compliant or "stiff" in a given application if its deflection under load from its equilibrium position can be neglected or if its dynamics (as characterized, for example, by its natural frequencies) are far from being excited by the range of frequencies required for its operation. A given actuator system may be compliant or not compliant depending on its use and the tasks assigned to it. There are two main sources of compliance, or in general impedance, in an actuator system. Active feedback control can modify the dyanmics of a system to give it the appearance of being compliant. In this case mechanical elements are not the source of the compliance (for example, see $[21,15,35])$. Alternatively passive mechanical elements can be used to introduce significant stiffness into the drive train. We are interested in the second method for this research [8].

\subsection{Benefits from the Use of Compli- ant Actuators}

Typical industrial robots are generally used in hot or hazardous environments, where heavy lifting is involved [2]. These robots are rigid and heavy which makes them unreasonable for many new applications. Low stiffness is important when a manipulator must interact with delicate or easily damaged objects, such as food. Also, when a robot is working in unstructured environments, compliance allows it to have controlled contact and interaction with rigid objects [23]. Stiff robots are not safe for interaction with human beings, due to the large, strong actuators used and the instabilities that can occur upon interaction, collision or failure of a component [29]. With elasticity, smooth rapid movements are possible and collisions are less serious due to the ability of the robot to absord energy in the springs, while without elasticity, robots are heavy and clumsy [20].

In several robotic applications direct interaction with humans is essential. The particular application used in this research is that of a prosthetic arm. In this application, size, weight and energy supplies are severely limited. Compliant actuators provide increased energy efficiency $[19,25,30,32]$ as well as reduced weight and cost [34]. Commercial prosthetic designs generally have not incorporated compliance, due to control challenges as well as space limitations. The few research based devices that do, however, have the goals of energy efficiency, low weight, and more natural movements [17]. A low stiffness prevents undesirable transmission of shock and vibration to the rest of the musculoskeletal system [14]. A critical goal of the amputee is to regain movement that appears "normal". It is, therefore, imperative for them to include muscle like, compliant actuators in order for them to move as naturally as possible $[6,7]$.

Compliance can be incorporated into actuators in a number of ways. The two central methods are with springs and with pneumatic actuators, both in series or in parallel. There are many designs using artificial muscles called McKibben, rubbertuators or Soft Arms, which are pneumatically driven [5]. Other methods used are hydraulic cylinders, fluidfilled bladders, pneumatic bladders, shape memory alloys [8] and ultrasonic motors [22]. Despite the above reasoning supporting the use of compliant robots, the lack of compliant actuators with acceptable performance and a lack of adequate controller architectures and computer techniques needed to take advantage of such actuators, means that progress is slow [28].

\subsection{Compliant Joints using Antago- nist Actuators}

Agonist-antagonistic actuators are located so that the agonist actuator acts to move the joint in one direction and the antagonist actuator acts in the opposite direction in a configuration analogous to muscles in a limb. This design is biologically inspired (eg. biceps and triceps) - generally the actuators need only act in tension and the net stiffness can be varied. Examples of these are found extensively in the previously mentioned areas of robotics, in arms, legs and fingers as well as in a limited number of prosthetic designs $[5,16,19,20,29,30,31]$. The main actuators used are springs and pneumatic artificial muscles, the most prominent being the pneumatic actuators. The design of the elbow prosthesis discussed in this paper uses antagonistically arranged, nonlinear springs as its compliant actuators. 
Antagonist actuators for a forearm prosthesis Koganezawa et. al. [17, 18] have done extensive research on using antagonistic muscle type actuators for use in robotics. Koganezawa has also applied this phenomenon to prosthetic limbs [16]. His earlier design uses AMAs (antagonistic muscle-like actuators) as an approach to imitating a skeletomuscular articulation system. It consists of 2 nonlinear conical springs, one DC motor which drives ball-screws and a pulley for the joint. Co-activation between the two nonlinear springs provides stiffness adjustment at the joint. For simplicity it is assumed that the springs are identical. Advantages of the antagonistic design include, a wide-range of joint stiffness regulation as well as reduction of backlash, an increased range of motion, wear compensation and the option of positioning the actuators remotely. The example application chosen was a forearm prosthesis with 6 degrees of freedom, 2 for the wrist and 4 for the fingers. It was concluded that simultaneous angular velocity feedback to both muscles, which is analogous to the stretch reflex in humans, is a successful method of damping [16]. A new elastic element is proposed in a second paper by Koganezawa called a NonLinear Elastic Module (NLEM) which overcomes drawbacks of the previous model. These include the difficulty to design specific nonlinear springs as well as the volume required to house the conical springs. NLEM is constructed with a torsion spring rolled around a guide rod which is aligned with the motor axis. A basic formula for controlling the stiffness and position of the end-point independently is derived and tested. It is found that the experimental results corresponded closely to theoretical results $[17,18]$.

Other applications based on compliant actuators include the design of orthotic devices [13]; improving the capabilities of walking robots $[24,33]$; a bionic robot arm driven by electric motors [20]; "CARL" which is a compliant articulated robot leg for dynamic locomotion [19]; and a biped walking robot $[31,32]$.

\section{Design Issues}

\subsection{Mechanics of Single Joints Driven by a Single Actuator}

Motor mechanics Electric motors operate by creating a rotating electromagnetic field. The overall performance of the motor is summarized in the torque constant (or the equivalent voltage constant) which relates the current in the windings to the torque generated between the stationery case (stator) and the rotating shaft (rotor). At this level the motor acts as a transformer that changes electric energy into mechanical energy. This operation is summarized in Figure 1 [4]. As shown in the figure the motor can either transmit power from the electrical side to the mechanical side (the region labeled "Motor") or can absorb mechanical power (the region labeled "Generator"). The power may be dissipated in the motor windings (Overhaul Type I) or transmitted out of the electrical port (Overhaul Type II). The practical limits (such as the thermal toque limit) can also be shown on this diagram. Plotting simulation results on a diagram such as Figure 1 can greatly aid in gaining an understanding of the demands placed on the motor and in choosing between various design alternatives. We have also extended the use of this diagram to include the additional complexities that result from the use of transmissions (both backdrivable and nonbackdrivable) [4].

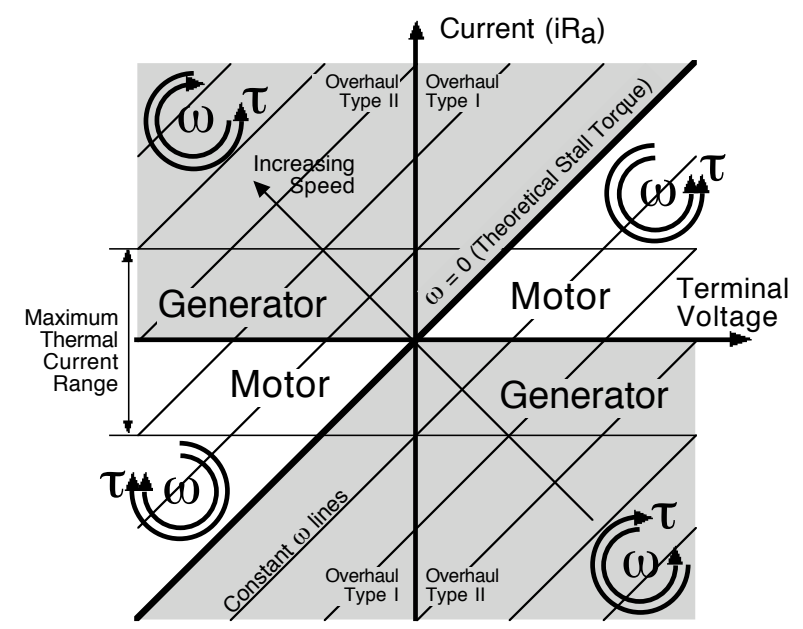

Figure 1: Basic Mechanics of an Electric Motor

At a more detailed level, the rotating electromagnetic field interacts with magnets (commonly) fixed to the body of the motor causing the motor to rotate. The natural dynamics of the motor fundamentally relate to the interaction of these two fields which behave like "virtual gear teeth". If a large torque is transmitted the two fields interact more strongly. There is, however, a maximum force that the fields will transmit which depends on the geometry. When the applied torque exceeds this value the "virtual gear teeth" slip. It is also possible to 
observe the effects of the interaction of these fields by carefully observing the torque output of a motor under load. Even with constant input current (meaning constant torque) a "torque ripple" that results from these "virtual teeth" approaching and separating from each other.

In almost all circumstances motors are chosen to have excess torque capabilities so that this slipping does not occur. In this case the dominant features of the motor dynamicss result from losses and inductance in the windings (typically a high frequency effect) and from the inertia of the rotating elements. However, in cases like prosthesis design where weight is a significant issue motors are often pushed to their limits and torques in excess of the maximum torque that can be sustained by the windings are encountered. The resulting "slipping" commonly results in a load squealing sound.

Using Feedback to Modify the Dynamics Limitations and Energetic Cost When it is necessary to modify the mechanical characteristics of a robot to improve its ability to interact with challenging environments the standard approach is to use position and/or force sensors to drive a feedback loop. In this way, a strong motor may appear to behave like a soft spring by, for example, measuring the position of the limb and responding with a force that is proportional to the deflection of the limb from a desired equilibrium position (a classical proportional controller). This approach is of course limited by the performance limitations of the motor but within these limitations the system characteristics can be altered without changing the physical design by modifying the controller constants. It is also achieved at the cost of increased complexity as sensors and control electronics must now be added to the device.

Another result of using feedback to modify the dynamic characteristics of a device is that there may be substantial energetic costs. For example, a real physical spring stores energy when is deflected, maintains a force on the disturbing agent without energetic cost and returns the original energy when the deflection is reduced. However, a robot that is controlled to appear to the environment as a spring requires energy to respond appropriately when it is deflected, requires a constant flow of energy to maintain a deflection, and requires further additional energy in order to return to its equilibrium position. These are real reasons to investigate what may be achieved by the use of physical springs to generate stiffness.

Gear Train Mechanics In order to gain this energy benefit using the physical springs shown in Figure 2 the motors must be able to lock their position to avoid expending the constant flow of energy required to maintain the desired force on the motor end of the spring. This may be achieved by the use of a clutch but in this case we have investigated the use of locking or nonbackdrivable gear sets to achieve this function. The classic nonbackdrivable gear set is the worm gear - worm wheel combination. However, no matter how this nonbackdrivable characteristic is achieved it is a result of the geometry of gear tooth contact and the level of friction. Therefore, in order to achieve the benefit of a steady state stiffness with zero energy cost the mechanism will have increased friction when it is in motion. A figure of merit has been developed that represents the ratio of time spent in static support of a load with low stiffness (with the transmission locked and the actuators turned off) to the time spent in motion [3]. The results show that if the time spent with the transmission locked is 1.93 times the time that the arm spends moving then there is an energetic benefit to this approach. Anecdotal evidence suggests that amputees spend a considerable amount of time using their prosthetic limbs as a stationary support and therefor this application may meet this criteria. However, as prosthetic limbs advance in dexterity this may change.

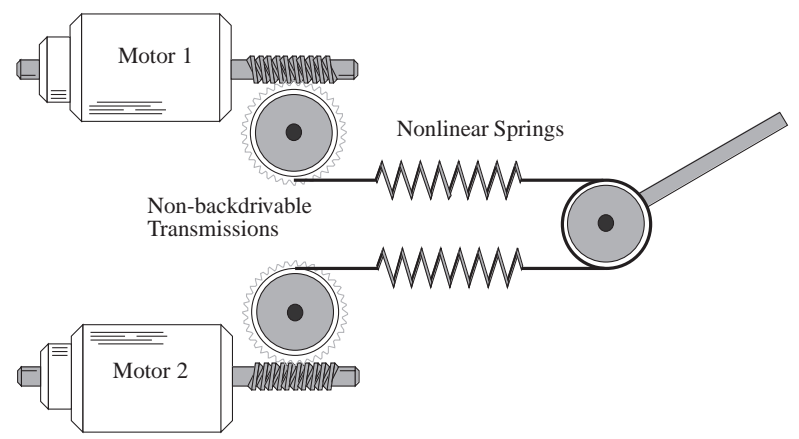

Figure 2: Basic Design Layout. A single motor with a feedback loop is replaced by two motors each placed in series with a stiffness element (spring). In some applications, a further advantage may be gained by using nonbackdrivable gear reductions on the motors so that power to the motors can be elliminated when the springs are all that is required to satisfy the performance requirements of the joint. 


\subsection{Mechanics of Single Joints Driven by an Agonist-antagonist Actua- tor Mechanics}

Figure 2 shows the basic configuration of two musclelike actuators acting around a joint. In this scenario there are now two actuators acting around a single degree of freedom. When both actuators act to move the joint in the same direction motion will occur but when the actuators act to produce equal and opposite torques the joint remains stationary. In this case the stiffness elements change length and, if they are nonlinear the net stiffness of the joint will change. Other configurations of springs and two actuators can be found that also allow this feature [8].

Net Joint Mechanics In a design situation it is necessary to develop criteria on which to base a selection of the nonlinear springs. Many are possible. From an analytical point of view one of the most appealing is to choose the spring stiffnesses so that the net stiffness of the joint is linear with respect to its angular displacement. It has been shown [10] that this is possible and that in order to achieve this the spring functions must be quadratic:

$$
\begin{aligned}
\phi(\delta)= & \frac{K_{M a x}-K_{M i n}}{4 R^{2} \delta_{P o s}} \delta^{2}+\frac{K_{M i n}}{2 R^{2}} \delta \\
& -\frac{\delta_{\text {pos }}\left(K_{\text {Max }}^{2}-2 K_{\text {Min }}^{2}\right)}{8 R^{2}\left(K_{\text {Max }}-K_{\text {Min }}\right)}
\end{aligned}
$$

In this equation, $K_{M a x}$ and $K_{M i n}$ are the maximum and minimum joint stiffnesses, $\delta_{\text {pos }}$ is the maximum stretch of the spring, $R$ is the radius of the line of action of the spring force about the center of rotation of the joint, and $\phi$ is the spring force which is a function of the deflection of the spring, $\delta$.

However, the need for this is not clear. In fact, from examining the properties of muscles, many seem to have stiffness characteristics that are far different from the "ideal" quadratic stiffness given in Equation 1. In using the quadratic stiffness elements defined here it is also critical to incorporate the real design limitations placed on performance due to the practical limits on the lengths of the springs and, depending on whether or not cables are used in the design, that the springs must remain in tension. If so, the range of deflections is constrained according to Figure 3. Care must be taken in working with the relationship between the individual spring stiffness functions and the net joint stiffness in an agonist - antagonist configuration as some conclusions that may seem intuitive are unfounded. For example, it is not necessary for the individual joint springs to be hardening (increasing in stiffness with deflection) for the net joint stiffness to be hardening.

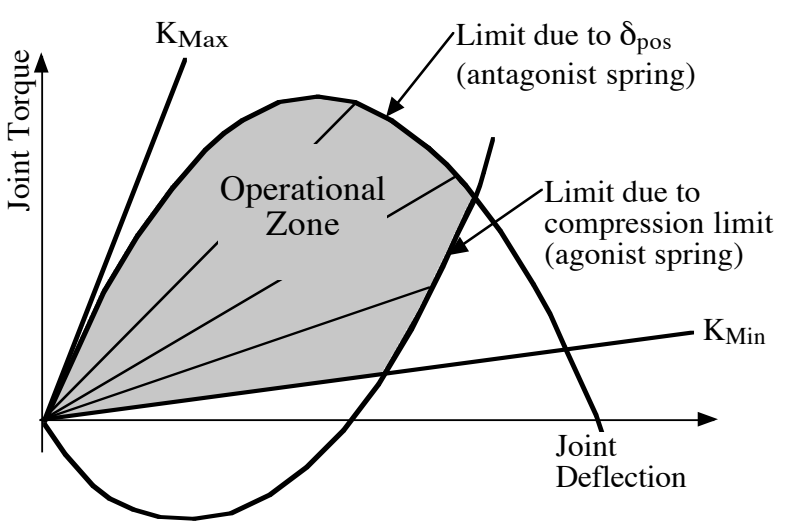

Figure 3: Range of Joint Operation as Limited by Deflection Limits on Quadratic Springs

Efficiency gains due to the use of a low stiffness during interaction. By using a configuration that allows the stiffness level to be effectively varied it is possible for the amputee (or user) to choose a level of stiffness in order to improve performance. In the case of interacting with a rigid movement constraint (for example opening a drawer) it has often been shown that it is energetically more efficient to apply forces in a direction in which no motion is allowed. This is a result of the fact that no work is done by these forces and that, in general, the most efficient direction in which a manipulator can generate a force does not correspond to a particular task. Commercial prosthetic limbs which generally have high levels of stiffness are incapable of taking advantage of this. In fact, it has been calculated that a limb with low stiffness may be on the order of 20 times more efficient at such tasks [25].

It is important to note that while the configuration explored in this paper is more similar to the biological case than the typical robotic drive, it is not identical. In the biological case the stiffness of a muscle changes with muscle activation. From a modeling point of view the force generating and stiffness elements are continuously distributed in the muscle fibre. An increase in muscle activation causes a resulting change in the properties of the 
muscle (including stiffness). As a result the net stiffness of the joint(s) about which the muscles act becomes changes (see Figure 4). The configuration in this paper achieves a change in stiffness by moving the equilibrium position of the spring. When the equilibrium positions of the springs are moved away from the joint they opreate in a region of higher stiffness (Figure 5).

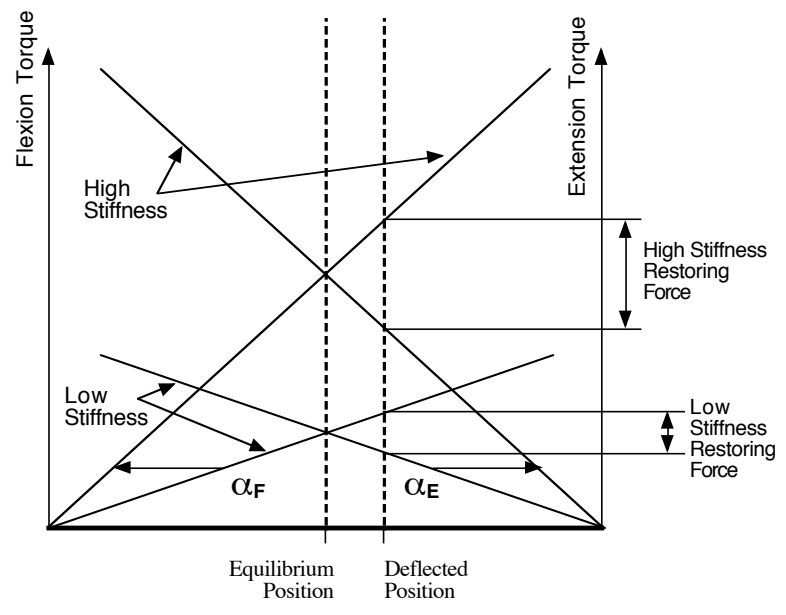

Figure 4: Coactivation of agonist-antagoinst muscles. When the muscle activation is increased the muscle stiffness (shown here as linear for simplicity) increases without a change in the equilibrium position of the muscle.

Limitations of the Design While this design is based on the biological model, it does not capture many of the features of the biological model that may be important. First, the actuator systems used in the design separate the energetic input (the motor) from the stiffness component (the spring). In the biological case these functions are effectively continuously distributed throughout a muscle. This means many of the particular mechanical properties of the muscle are not captured in this design. Further, it is also interesting to note that the energy flows are simple in the proposed design while in a muscle complex biochemical processes mean that the same amount of metabolic energy does always produce the same level of muscular work.

The chosen design does have some benefits over the typical robotic approach. By using real springs it is no longer required that the motor be backdrivable. Large forces (including impact forces) have

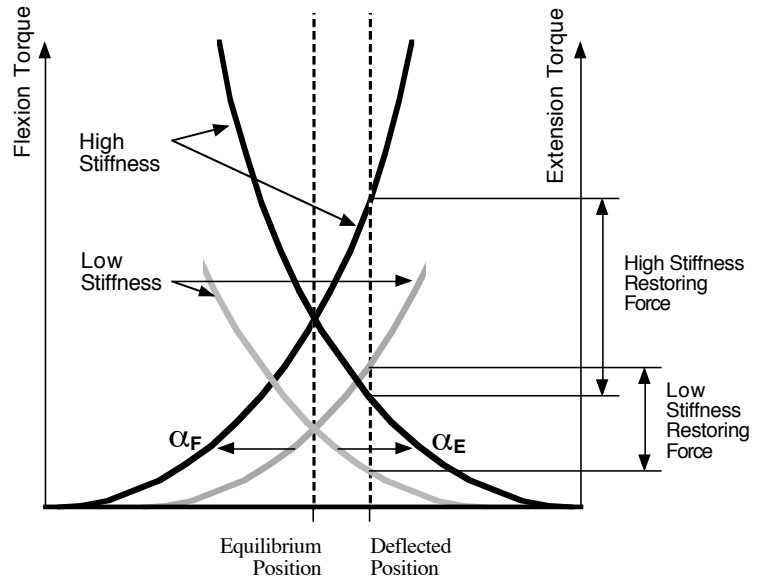

Figure 5: Prosthesis Design. By increasing the force generated by each motor the stiffness changes as a result of a change in the equilibrium position of the nonlinear stiffness element.

a minimal effect on the motor as the energies that are involved can be stored / supplied from the conservative physical elements in the system (including in a dynamic situation, the mass of the limb). An actuator system that is backdrivable generally uses a large high-torque, direct-drive motor or uses a smaller higher speed motor with a large gear reduction. Limitations in performance in the robotic case arise from the generally larger size of a torque motor or from the limitations on gear trains that remain backdrivable. In this biomimetic design, a high speed motor may be used with a large gear ratio reduction and still present a low stiffness to the environment.

\section{Mechanics of Limbs with Com- pliant Joints}

When this type of actuator design is proposed for use in a multi-joint manipulator several new issues arise. In the case of using this design for an elbow prosthesis there are several significant differences between the amputated limb / prosthesis combination and a healthy limb. The focus of this research has been on the relationship between stiffnesses in various parts of the system. The particular concern in the elbow prosthesis was to determine if the stiffness effects of the intact limb could be duplicated by the limb-prosthesis combination despite the lack of the two joint muscles (biceps and triceps). 
A detailed analysis of the system shown in Figure 6 was performed. The results indicated that in general the intact limb with the two-joint muscles could generate a greater range of possible stiffnesses at the hand than could the limb with the prosthesis [11]. In the process of performing this analysis several interesting relationships between the actuator stiffnesses and the limb stiffnesses were discovered. In particular, a method for representing the two-joint stiffnesses as an equivalent single joint stiffness was found [27] (see Figure 7).

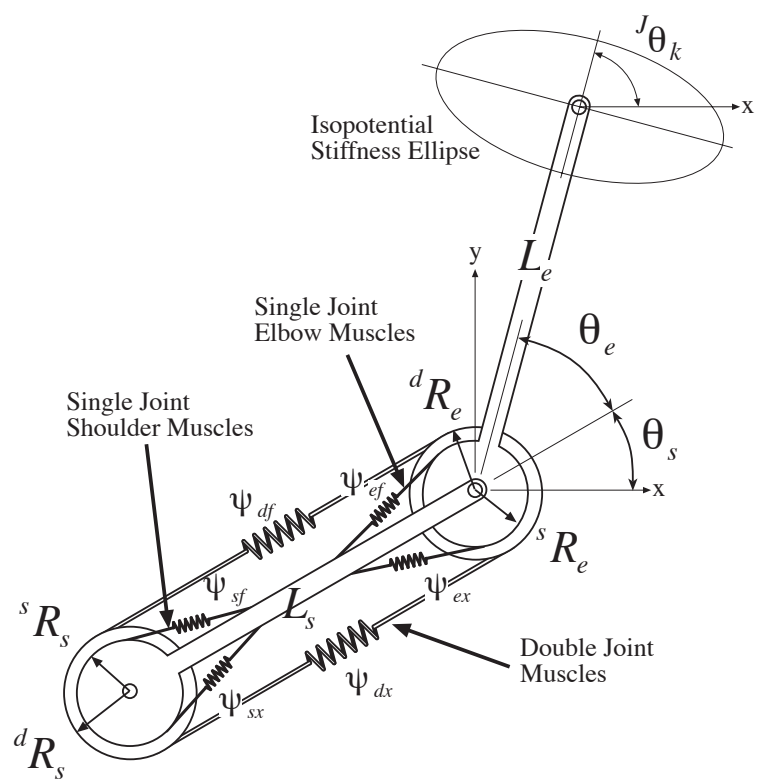

Figure 6: Stiffnesses in the model limb. The stiffness of the hand was investigated in terms of six individual stiffness elements placed in positions analogous to the primary muscles involved in arm motion.

Another interesting result of the analysis relates to the contribution to the overall "hand" stiffness from geometric effects $[12,9]$. The stiffness of the hand can be written as:

$$
\begin{aligned}
\mathbf{K} & =\left(\begin{array}{ll}
\frac{\partial F_{x}}{\partial x} & \frac{\partial F_{x}}{\partial y} \\
\frac{\partial F_{y}}{\partial x} & \frac{\partial F_{y}}{\partial y}
\end{array}\right) \\
& =\mathbf{J}^{-T} \mathbf{\Psi} \mathbf{J}^{-1}+\frac{d\left(\mathbf{J}^{-T}\right)}{d \theta} \mathbf{M} \mathbf{J}^{-1}
\end{aligned}
$$

In this equation, $\mathbf{K}$ is the stiffness matrix; $F_{x}, F_{y}$, $x$ and $y$ are the vector components of the endpoint force and displacement; $\mathbf{J}$ is the Jacobian matrix;

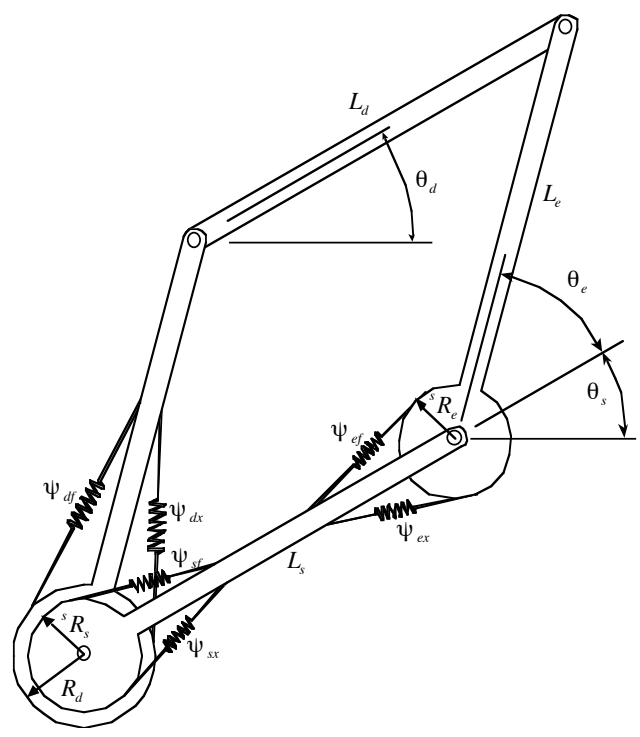

Figure 7: A four bar linkage with equivalent "hand" stiffness to a limb. This linkage does not require two-joint stiffness elements.

$\boldsymbol{\Psi}$ is a matrix containing the stiffnesses of the individual stiffness elements; $\theta$ is a vector containing the joint angles and $\mathbf{M}$ is a vector of joint moments. The second term in the equation represents the effects of changing geometry on the joint stiffness. It can, under reasonable choices of position and force that correspond to activities commonly performed by the human limb, be unstable. In fact, it can contribute enough unstable stiffness to the overall stiffness, $\mathbf{K}$, that the limb becomes unstable and will move to an alternate equilibrium position.

\section{A Biomimetic Controller}

One of the major areas of future work in this project is to clarify appropriate methods of controlling robots and prosthetic limbs with multiple redundant controllers. The ability of the human arm to interact with complex unknown dynamic environments demonstrates that solutions to this control problem that do not rely on detailed knowledge or measurement of the dynamic environment are possible. Preliminary work on a controller for this type of system [26] is based on an interpretation of the basic neural system used to control muscles in the human arm. A schematic of this layout is shown in Figure 8 


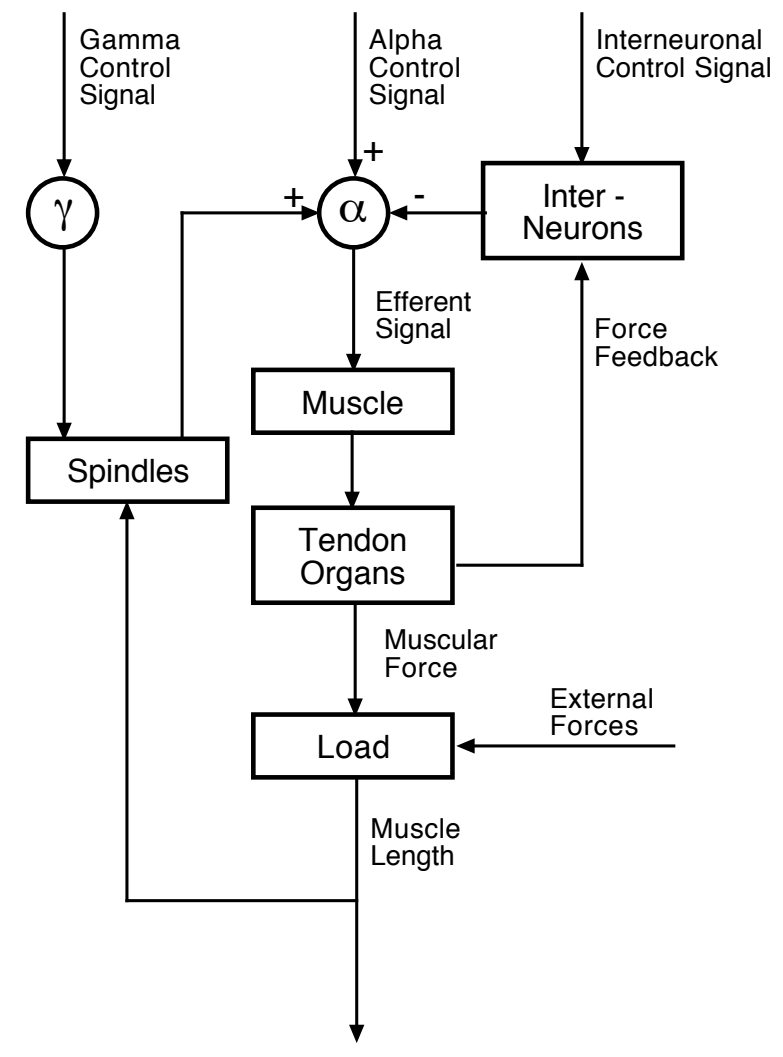

Figure 8: A block diagram summarizing the neural pathways for controlling skeletal muscle.
From an engineering point-of-view there are a number of interesting features to this configuration. One is the duplication of control input signals (alpha and gamma). The usual explanation is that the gamma control signal is there to keep the spindle at it's optimal length for measuring the muscle kinematics. In developing a biomimetic approach to the control of systems with multiple, redundant, compliant actuators a novel interpretation of this network was adopted. Based on the assumption that the body would not use two separate nerve signals when one would be sufficient, we surmised that the gamma signal may contain an intentional perterbation from the main alpha control signal. The spindle fibres contribute little to the overall force of the muscle so the effect of this perterbation on performance would be minimal. With this perterbation in place we hypothesize that the signal returning from the spindle would contain information on any benefits that might have been achieved by the perturbed signal. Simulations of this type of control system have been performed and show that it can be structured so that the efferent signal has a continual tendency to reduce the muscle output and that feedback from the results of the perturbed signal successfully reinforces the perturbations which actually increase the work done by the muscle. In other words, this architecture attempts to use the perturbed signal to provide information that allows for a local optimization of the work done by the muscle. The simulations show that in the presence of an external agent or other actuators, the work done by the muscle is reduced. Significantly more work on this control architecture is the topic of future research efforts.

\section{Conclusions}

This work on applying biomimetic design approaches to the design of prosthetic limbs has generated results in a number of areas. A novel design for a prosthetic limb with many promising features and improved performance has been developed. Issues addressed in the design have provided insight into an increased understanding of the contributions of two-joint muscles, particularly in terms of the overall limb stiffness. The existence of unstable configurations for the limb as a result of the geometric components of stiffness suggest that systems like this, perhaps including the human limb, may not be stable without an active control system. Finally a novel approach to controlling these systems has been proposed. 


\section{Acknowledgments}

The authors acknowledge funding from the Natural Sciences and Engineering Research Council of Canada and the support of Carleton University.

\section{References}

[1] Beer, R.D., Chiel, H.J., Quinn, R.D., Ritzmann, R.E. Biorobotic Approaches to the Study of Motor Systems. Current Opinion in Neurobiology 8(6): 777-782 (1998).

[2] Boubekri, N., Chakraborty, P. Robotic grasping: gripper designs, control methods and grasp configurations a review of research. Integrated Manufacturing Systems 13(7): 520-531 (2002).

[3] T. Burke and D. L. Russell Benefits of using Passive Stiffness Elements in Interacting Prosthetic Limbs - A Figure of Merit in Proceedings of CMBEC 26, October 2000.

[4] T. Burke Modelling and Evaluation of Nonbackdrivable Transmissions for Variable Stiffness Prostheses. Masters Thesis, Carleton University, Ottawa, Ontario, April, 2000.

[5] Caldwell, D.G., Tsagarakis, N. Soft grasping using a dextrous hand. Industrial Robot: An International Journal 27(3): 194-199 (2000).

[6] Cheng, G., Nagakubo, A., Kuniyoshi, Y. Continous humanoid interaction: An integrated perpective gaining adaptivity, redundancy, flexibility in one. Robotics and Autonomous Systems 37: 161183 (2001).

[7] Dario, P., Guglielmelli, E., Laschi, C. Humanoids and Personal Robots: Design and Experiments. Journal of Robotic Systems 18(12): 673-690 (2001).

[8] C. English. Mechanics and Design of a Variable Stiffness Actuator for uses in Impedance Control. Masters Thesis, Carleton University, Ottawa, Ontario, April,1996.

[9] C. English Stiffness Behaviour in Two Degree of Freedom Mechanisms Ph. D. Thesis, Carleton University, Ottawa, Ontario, May, 2000.

[10] C. English and D. Russell, Mechanics and Stiffness Limitations of a Variable Stiffness Actuator for Use in Prosthetic Limbs, in Mechanism and Machine Theory, 1999 Jan. Vol.34 No.1 Pages 7 $-25$

[11] C. English and D. Russell, The Influence of Double Joint Muscles on Interactive Stiffness, in proceedings of CMBEC 25, June 1999, pgs 98-99.
[12] C.E. English and D.L. Russell, An Approach to Analysing Unstable Planar Manipulator, in proceedings of DYCON 1999, Ottawa Ontario, 8 pages.

[13] Harwin, W.S. Design and control of a compliant mobile arm support for assisting arm movements. Advancement of Assistive Technologies 3: 320-325 (1997).

[14] Hogan, N. Adaptive Control of Mechanical Impedance by Coactivation of Antagonist Muscles. IEEE Transactions on Automatic Control AC29(8) (1984).

[15] Hogan, N., Impedence Control: An Approach to Manipulation: Part 1 Theory, Part 2 Implementation, Part 3 Applications. Journal of Dynamic Systems, Measurement and Control 107:124 (1985).

[16] Koganezawa, K., Watanabe, Y., Shimizu, N. Antagonist muscle-like actuator and its application to multi-d.o.f. forearm prosthesis. Advanced Robtics 12(7): 771-789 (1999a).

[17] Koganezawa, K., Yamazaki, M. Mechanical Stiffness Control of Tendon-Driven Joints. Preceedings of the 1999 IEEE/RSJ International Conference on Intelligent Robots and Systems (1999b).

[18] Koganezawa, K., Ban, S. Stiffness Control of Antagonistically Driven Redundant D.O.F. Manipulator. Proceedings of the 2002 IEEE/RSJ International Conference on Intelligent Robots and Systems (2002).

[19] Mennitto, G., Buehler, M. CARL: A compliant articulated robot leg for dynamic locomotion. Robotics and Autonomous Systems 18: 337-344 (1996).

[20] Moehl, B. A Bionic Robot Arm with Compliant Actuators. Proceedings of SPIE 4196: 82-85 (2000).

[21] Park, J.H. Impedance Control for Biped Robot Locomotion. IEEE Transactions on Robotics and Automation 7(6) (2001).

[22] Pecson, M., Ito, K., Luo, Z., Kato, A., Aoyama, T., Ito, M. Compliance Control of an Ultrasonic Motor Powered Prosthetic Forearm. IEEE International Workshop on Robot and Human Communication (1993).

[23] Pfreundschuh, G.H., Kumar, V., Sugar, T. Design and Control of a 3 DOF in-Parallel Actuated Manipulator. Proceedings of the 1991 IEEE International Conference on Robotics and Automation (1991). 
[24] Quinn, R.D., Nelson, G.M., Bachmann, R.J., Kingsley, D.A., Offi, J., Ritzmann, R.E. Insect Designs for Improved Robot Mobility. Proceedings of the 4th International Conference on Climbing and Walking Robots, Berns and Dillmann eds., Prof. Eng. Pub., 69-76 (2001).

[25] Russell, D.L., Energy Expenditure in Upper Limb Prostheses Performing Constrained Movements. UNB's International Symposium on Myoelectric Control, 1993.

[26] Z. Sheng A Novel, Neuro-Muscular, Actuator Control Scheme. Masters Thesis, Carleton University, Ottawa, Ontario, September, 1997.

[27] D. Russell and C. English An Equivalent Linkage Model for Understanding Stiffness Effects of Biarticular Muscles , ASME 2001 International Mechanical Engineering Congress and Exhibition, Nov., 2001, 2 pages.

[28] Tamura, Y., Saito, M. Adaptive Compliant Motion Control of Pseudo-Musculat Visco-Elastic Actuator: Simulation and Experiments. 11th Conference of the ESB (1998).

[29] Tuijthof, G.J.M., Herder, J.L. Design, actuation and control of an anthropomorphic robot arm. Mechanism and Machine Theory 35: 945-962 (2000).

[30] van der Linde, R.Q. Design, Analysis, and Control of a Low Power Joint for Walking Robots by Phasic Activation of McKibben Muscles. IEEE Transactions on Robotics and Automation 15(4) (1999).

[31] Yamaguchi, J., Takanishi, A. Development of a Biped Walking Robot Having Antagonistic Driven Joints Using Nonlinear Spring Mechanism. Proceedings of the 1997 IEEE International Conference on Robotics and Automation (1997).

[32] Yamaguchi, J., Nishino, D., Takanishi, A. Realization of Dynamic Biped Walking Varying Joint Stiffness Using Antagonistic Driven Joints. Proceedings of the 1998 International Conference on Robotics and Automation (1998).

[33] Yi, K.Y. Locomotion of a biped robot with compliant ankle joints. Proceedings of the 1997 IEEE International Conference on Robotics and Automation (1997).

[34] Yi, K.Y. Walking of a Biped Robot with Compliant Ankle Joints: Implementation with KUBCA. Proceedings of the 39th Conference on Decision and Control (2000).
[35] Zollo, L., Siciliano, B., Laschi, C., Teti, G., Dario, P. Compliant control for a cable- actuated antropomorphic robot arm: An experimental validation of different solutions. Proceedings of the 2002 IEEE International Conference on Robotics and $\mathrm{Au}-$ tomation (2002). 